Published in J. Phys. A: Mat. Gen. 26, L385-390 (1993).

\title{
STOCHASTIC RESONANCE IN MONOSTABLE SYSTEMS
}

\author{
N G Stocks, N D Stein and P V E McClintock \\ School of Physics and Materials, \\ Lancaster University, \\ Lancaster, LA1 4YB, \\ U.K.
}

\begin{abstract}
$\underline{\text { Abstract }}$
The first observations of noise-induced enhancements and phase-shifts of a weak periodic signal - characteristic signatures of stochastic resonance (SR) - are reported for a monostable system. The results are shown to be in good agreement with a theoretical description based on linear response theory and the fluctuation dissipation theorem. It is argued that SR is a general phenomenon that can in principle occur for any underdamped nonlinear oscillator.
\end{abstract}

PACS: $05.40+\mathrm{j}$, 78.50.-w, 05.20.Dd 
Stochastic resonance (SR) is widely perceived as a phenomenon peculiar to bistable systems in which, under appropriate conditions, a weak periodic signal can be amplified by the introduction of external noise. It has been studied in a variety of contexts including meteorology [1], lasers [2], passive optical systems [3], electron spin resonance (ESR) [4], electronic circuits $[5,6]$ and a magnetoelastic ribbon [7]; arguably it is also of relevance to the function of sensory neurons [8]. In all of these cases, the mechanism [9] of stochastic amplification depends on the onset, for appropriate noise intensity, of quasi-periodic fluctuational transitions between coexisting attractors corresponding to the minima of a static bistable potential. At first sight, therefore, it would appear that there are powerful a priori reasons to suppose that SR cannot occur at all unless the system under study possesses at least two coexisting attractors; but we will demonstrate below that the conventional wisdom is wrong. In this Letter we develop a rather different and much more general perception of SR that shows, remarkably, that the phenomenon need not be confined to static bistable (or multistable) systems but can also manifest itself, for example, in a class of monostable nonlinear oscillators.

We propose that candidate systems in which to seek new forms of SR may readily be identified through the perception of SR as a linear response phenomenon [6]. In practice, one should seek systems which, when driven by Gaussian white noise, exhibit one or more well-defined sharp maxima in the spectral densities $Q_{0}(\omega)$ of their fluctuations (SDFs) in the absence of the periodic force. In those cases where the SDF close to such a maximum increases rapidly with noise intensity $T$, the fluctuation dissipation theorem [10] implies that the susceptibility of the system must also be increasing with $T$, and a manifestation of SR is therefore to be expected when the weak periodic force is added to the system. (In this scenario, conventional SR is associated with the zero-frequency SDF peak [15] 
corresponding to inter-well hopping within the static bistable potential.)

The advantages and disadvantages of the linear response theory (LRT) approach to SR have already been rehearsed elsewhere [11]. Nonetheless, it may be worth repeating that the disadvantage of LRT is that it is only applicable when (as is often the case in practice) the periodic force is weak. Of its many advantages we would emphasize here only that, in contrast to other theories of SR (e.g. [12-14]), LRT avoids the problem of non-stationarity by calculating the susceptibility from $Q_{0}(\omega)$ in the absence of the periodic force.

An example of a promising monostable candidate system in which to seek SR, which fulfills [16] the criteria proposed above, is provided by the single-well Duffing oscillator driven by Gaussian white noise plus a weak periodic force

$$
\begin{gathered}
\ddot{q}+2 \Gamma \dot{q}+\frac{d U(q)}{d q}=f(t)+A \cos \Omega t \\
U(q)=\frac{1}{2} q^{2}+\frac{1}{4} q^{4}+B q \\
\Gamma \ll 1, \quad\langle f(t)\rangle=0 \\
\left\langle f(t) f\left(t^{\prime}\right)\right\rangle=4 \Gamma T \delta\left(t-t^{\prime}\right)
\end{gathered}
$$

We consider two distinct cases, depending on the magnitude of $|B|$. In case (a), $|B|<$ 0.43, the variation of the oscillator's eigenfrequency $\omega(E)$ with energy $E$ measured from the bottom of the potential well is monotonic [16], as sketched in Fig 1(a). In the absence of the periodic force $(A=0)$, for small noise intensity $T$, a narrow Lorentzian peak of width $\sim \Gamma$ occurs in the SDF at frequency $\omega(0)$. As $T$ is increased, the average energy $\bar{E}$ of the 
oscillator rises, and the peak broadens asymmetrically [16] towards higher frequencies. For an $\Omega>\omega(0)$, initially on the tail of the peak i.e. $[\Omega-\omega(0)]>\Gamma$, the magnitude of $Q_{0}(\Omega)$ therefore increases very rapidly (approximately exponentially) with $T$. The corresponding increase in the generalised susceptibility $\chi(\Omega)$ of the system implied by the fluctuation dissipation theorem [10] means, in turn, that a weak periodic force on the right hand side of (1) will be amplified by an increase of $T$. Surprising though it may seem at first sight, SR is therefore to be anticipated in (1) even though the system is monostable, with $U(q)$ a smooth single-well potential. The resonance maximum is to be expected when $T$ has been "tuned" to adjust $\bar{E}$ such that $\omega(\bar{E}) \sim \Omega$. This argument is extremely general and can obviously be applied, perhaps with minor variations, to any underdamped nonlinear oscillator.

In case (b) with $|B|>0.43$, on the other hand, $\omega(E)$ is nonmonotonic [16], as sketched in Fig $1(\mathrm{~b})$. In the absence of the periodic force $(A=0)$, the system exhibits noiseinduced spectral narrowing [16]; and, for sufficiently small values of the damping constant $\Gamma$, exceedingly sharp zero-dispersion spectral peaks (ZDPs) of width $\propto \Gamma^{\frac{1}{2}}$ appear [17] close to the frequency $\omega_{m}$ of the extremum where $d \omega(E) / d E=0$. The magnitude of the ZDP rises exponentially with increasing $T$. Just as in case (a) the corresponding rapid increase of $\chi(\Omega)$ implies a manifestation of SR for $\Omega$ close to $\omega_{m}$. The extreme narrowness of the ZDP [17] suggests that SR in case (b) will be a much more dramatic phenomenon than in case (a). [Clearly, a case (a) type resonance can also be obtained by making $\Omega>\omega(0)$, even when $|B|>0.43$.]

To test these predictions, we have sought evidence of SR in an electronic model of (1) designed, constructed, and operated according to standard practice [18]. For the results to be presented below, the parameter values used were: $\Gamma=0.011 ; A=0.020 ;$ and $B=$ 
0 or $B=2.00$ for cases (a) or (b) respectively. The value of $\Gamma$ was in fact too small to be determined directly from the circuit components (owing to the effect of stray capacitance and other non-idealities); instead, it was measured in a separate experiment. The model was driven with quasi-white noise from an external noise-generator, and with a weak periodic force from an HP3325 frequency synthesizer. The resultant fluctuating $q(t)$ was digitized in 1024 point blocks and ensemble-averaged by a Nicolet LAB80 data-processor to yield $\langle q(t)\rangle$. The advantage of averaging in the time (rather than the frequency) domain is that it enables measurements to be made of the phase shift $\phi$ between the drive and the response $[4,6,11]$ as well as yielding directly the amplitude $a$ of the response. Measurements were made of the stochastic amplification factor [19]

$$
S(T)=a(T) / a(0)
$$

and of the corresponding value of $\phi$ over a wide range of $T$ for the two cases (a) and (b).

Some typical measurements of the noise-induced power gain are shown by the data points in Fig 2 (where we plot $S^{2}$, rather than $S$, for more convenient comparison with earlier SR results). It is immediately evident that, both for case (a) (squares) and for case (b) (circles), $S^{2}$ at first increases rapidly with noise intensity, but then passes through a maximum and decreases again, albeit more slowly. That these data should bear a striking resemblance to those obtained previously for conventional SR [2-7] is, of course, no coincidence. We emphasize that, in the case of a linear system (harmonic oscillator), $S^{2}$ would not increase at all but would remain equal to unity irrespective of the value of $T$. As anticipated, the maximum is much larger for case (b) than for case (a); it can be shown [20] that, in case (b) for sufficently small $\Gamma$, not only the signal but also the signal/noise ratio increases with increasing $T$ within a certain range. 
The measured phase lag $-\phi$ between the drive and response is plotted for case (a) (squares) and case (b) (circles) in Fig 3. The forms of $-\phi(T)$ for the two cases are strikingly different, but they can readily be understood qualitatively by analogy with a conventional (deterministic) resonance. In case (a) for $T=0$, the periodic driving force is being applied at a frequency well beyond the natural frequency of the system, $\Omega>\omega(0)$ [see Fig 1(a)]. Consequently $-\phi$ is close to $180^{\circ}$. As $T$ is increased, however, the "natural frequency" $\omega(E)$ is effectively being tuned past the fixed driving frequency. Near resonance $-\phi$ passes through $90^{\circ}$ and, in the high $T$ limit where the "natural frequency" substantially exceeds $\Omega,-\phi$ decreases towards $0^{\circ}$ exactly as would be seen in a conventional resonance. The phase changes for case (b), while quite different, can be accounted for in a closely similar way. In this case, the "natural frequency" $\omega(E)$ always exceeds that of the drive $\Omega$ [Fig $1(\mathrm{~b})]$, and so the phase lag $-\phi$ is always less than $90^{\circ}$, although it approaches $90^{\circ}$ near the resonance maximum, just as expected.

A quantitative theoretical description of these phenomena is readily constructed on the basis of linear response theory (LRT), and is relatively straightforward given that the SDF of (1) for $A=0, Q_{0}(\omega)$, is already known [16]. The susceptibility $\chi(\Omega)$ of the system can be found immediately from the fluctuation dissipation theorem [10]

$$
\begin{gathered}
\operatorname{Re} \chi(\Omega)=\frac{2}{T} \mathrm{P} \int_{0}^{\infty} d \omega_{1}\left[\omega_{1}^{2} /\left(\omega_{1}^{2}-\Omega^{2}\right)\right] Q_{0}\left(\omega_{1}\right) \\
\operatorname{Im} \chi(\Omega)=(\pi \Omega / T) Q_{0}(\Omega)
\end{gathered}
$$

where $\mathrm{P}$ implies the Cauchy principal part. The squared amplitude of the response is then just $a^{2}=A^{2}|\chi(\Omega)|^{2}$ so that 


$$
S^{2}=[a(T) / a(0)]^{2}=|\chi(\Omega)|^{2} /\left\{\left[\omega(0)^{2}-\Omega^{2}\right]^{2}+4 \Gamma^{2} \Omega^{2}\right\}
$$

and

$$
\phi=-\tan ^{-1}[\operatorname{Im} \chi(\Omega) / \operatorname{Re} \chi(\Omega)]
$$

The quantity $Q_{0}(\omega)$ in (3) and (4) - which, we emphasize again, is the SDF in the absence of the periodic force - was obtained from Eq (20) of Ref [16], also using its Eqs (4a), 4(b), (14), (A7), (A9) and a numerical solution of its (16). Values of $S^{2}$ and $\phi$ calculated in this way from (3)-(6) for (1) with the parameters used in the circuit and are plotted (full curves) as functions of $T$ in Figs 2 and 3 for comparison with the experimental measurements. Given that there are no adjustable parameters, the agreement between experiment and theory can be regarded as excellent.

The physical mechanisms of these stochastic amplification phenomena differ radically from that of conventional SR [9], and it might therefore be argued that the new effects reported in this Letter deserve to be given an entirely different name. We would disagree with any such proposition, however, on two grounds: because, as we have shown, a unified theoretical description of both sets of phenomena can be constructed in terms of LRT and the fluctuation dissipation theorem; and also because, ironically, the term stochastic resonance actually provides an apter description of the new phenomena than it does of conventional SR which, strictly, should not [21] be referred to as a resonance at all.

In conclusion we would emphasize, first, that the case (a) variant of SR discovered and investigated in the present work is in no way confined to the particular system (1). Rather, it is a quite general phenomenon, to be anticipated in all underdamped nonlinear oscillators. In all situations where, as in case (b) the eigenfrequency has a smooth 
extremum in its variation with energy (or has a higher singularity), a more pronounced manifestation of $\mathrm{SR}$ is to be anticipated: it might reasonably be called zero-dispersion stochastic resonance (ZDSR). Secondly, there is an interesting distinction between the present results and those of conventional SR. In the latter case, stochastic amplification occurs in both overdamped and underdamped systems but is at its most pronounced when the damping is large; the new forms of SR studied above, on the other hand, are entirely restricted to underdamped systems and are most pronounced when the damping is small. Finally, the differing dependences of the SR phase shift on noise intensity for cases (a) and (b) have helped to illuminate in a satisfying way the remarkably close analogy that exists between stochastic and deterministic resonance phenomena.

This work developed out of discussions with Mark Dykman, who first proposed that SR should be considered within the conceptual framework provided by LRT and the fluctuation dissipation theorem, with Riccardo Mannella who drew our attention to the significance of phase shifts in SR, and with Slava Soskin who predicted the existence of the zero dispersion spectral peaks. It was supported by the Science and Engineering Research Council (UK), by the Royal Society of London, and by the European Community. 


\section{REFERENCES}

1. R Benzi, A Sutera and A Vulpiani, J. Phys. A 14, L453 (1981); C Nicolis, Tellus

34, 1 (1982); R Benzi, G Parisi, A Sutera and A Vulpiani, Tellus 34, 10 (1982).

2. B McNamara, K Wiesenfeld and R Roy, Phys. Rev. Lett. 60, 2626 (1988).

3. M I Dykman, A L Velikovich, G P Golubev, D G Luchinsky and S V Tsuprikov, Sov. Phys. J.E.T.P. Lett. 53, 193 (1991).

4. L Gammaitoni, M Martinelli, L Pardi and S Santucci, Phys. Rev. Lett. 67, 1799 (1991).

5. S Fauve and F Heslot, Phys. Lett. 97A, 5 (1983); L Gammaitoni, F Marchesoni, E Menichella-Saetta and S Santucci, Phys. Rev. Lett. 62, 349 (1989).

6. M I Dykman, P V E McClintock, R Mannella and N G Stocks, Sov. Phys. J.E.T.P. Lett. 52, 141 (1990); M I Dykman, R Mannella, P V E McClintock and N G Stocks, Phys. Rev. Lett. 65, 2606 (1990).

7. M L Spano, M Wun-Fogle and W L Ditto, Phys. Rev. A 46, 5253 (1992).

8. A Longtin, A Bulsara and F Moss, Phys. Rev. Lett. 67, 656 (1991).

9. B McNamara and K Wiesenfeld, Phys Rev A 39, 4854 (1989).

10. L D Landau and E M Lifshitz, Statistical Physics, 3rd edn, (Pergamon, New York, 1980).

11. M I Dykman, R Mannella, P V E McClintock and N G Stocks, Phys. Rev. Lett. 68, 2985 (1992). 
12. C Presilla, F Marchesoni and L Gammaitoni, Phys. Rev. A 40, 2105 (1989).

13. Hu Gang, G Nicolis and C Nicolis, Phys. Rev. A 42, 2030 (1990).

14. P Jung and P Hanggi, Phys. Rev. A 44, 8032 (1991).

15. M I Dykman, R Mannella, P V E McClintock, F Moss and S M Soskin, Phys. Rev. A 37, 1303 (1988).

16. M I Dykman, R Mannella, P V E McClintock, S M Soskin and N G Stocks, Phys. Rev. A 427041 (1990).

17. S M Soskin, Physica A 155, 401 (1989); and Physica A 180, 386 (1992).

18. L Fronzoni in Noise in Nonlinear Dynamical Systems, ed F Moss and P V E McClintock (CUP, Cambridge, 1989) vol 3, p 222; P V E McClintock and F Moss in op. cit. p 243; N G Stocks, Experiments in Stochastic Nonlinear Dynamics, PhD thesis, Lancaster, 1990 (unpublished).

19. In the spirit of [14], we discuss the SR effect in terms of the noise-induced amplification of the signal, rather than that of the signal/noise ratio as used in many earlier papers on SR. The stochastic amplification factor $S$ defined by (2) has the convenient feature that an absence of stochastic amplification is characterised by $S$ $=1$.

20. N G Stocks, N D Stein, S M Soskin and P V E McClintock, J Phys A 25, L1119 (1992).

21. R F Fox, Phys. Rev. A 39, 4148 (1989). 


\section{FIGURES}
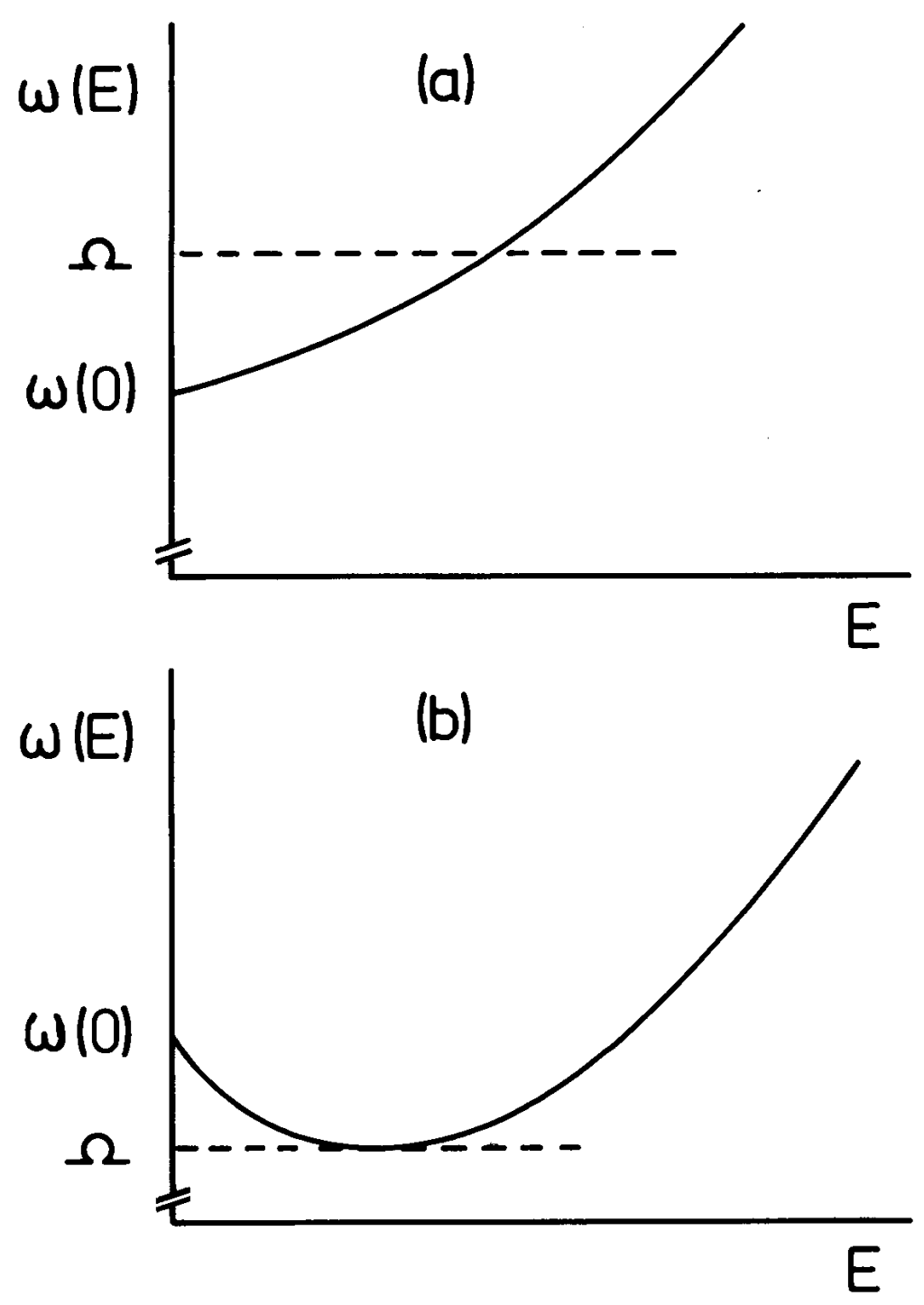

Figure 1: Sketches to show the dependence of the eigenfrequency $\omega(E)$ on energy $E$ measured from the bottom of the potential well for the system (1): (a) for $|B|<0.43$; (b) for $|B|>0.43$. Frequencies $\Omega$ at which a weak periodic force will be amplified by SR are indicated. 


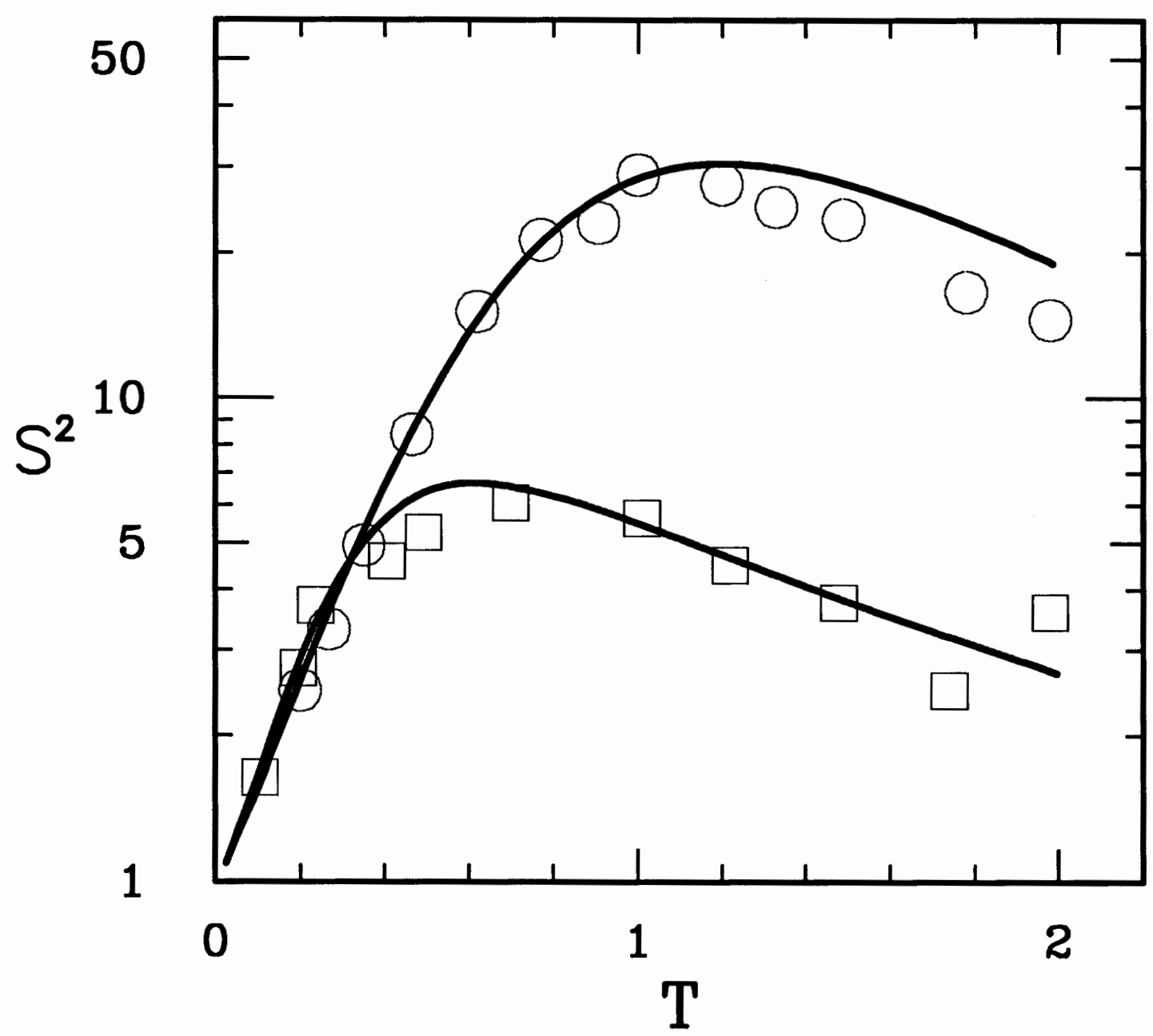

Figure 2: The squared stochastic amplification factor $S^{2}$ measured for case (a) (squares) and case (b) (circles) as a function of noise intensity $T$ for the electronic circuit model of (1) with $A=0.02$ is compared with the theoretical predictions (full curves) obtained from the fluctuation dissipation theorem. 


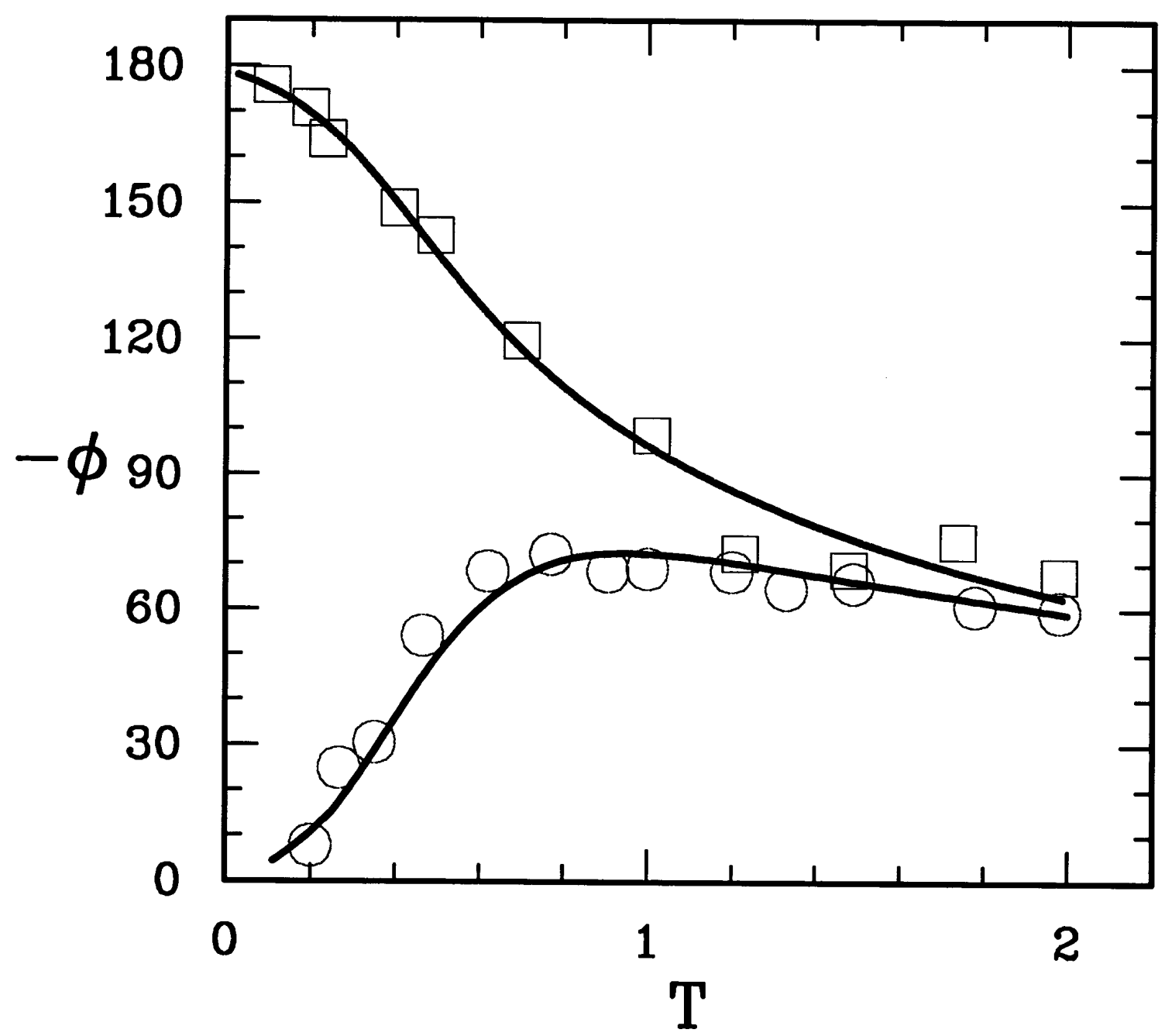

Figure 3: The phase difference $-\phi$ (in degrees) between the drive $B \cos \Omega t$ and the response $\langle q(t)\rangle$ measured for case (a) (squares) and case (b) (circles) as a function of noise intensity $T$ for the electronic circuit model of (1) with $A=0.02$ is compared with the theoretical predictions (full curves) obtained from the fluctuation dissipation theorem. 